\title{
Detection of cholesteatoma: High-resolution DWI using RS-EPI and parallel imaging at 3 tesla
}

\author{
O. Algin ${ }^{\mathrm{a}, \mathrm{b}, *}$, H. Aydın ${ }^{\mathrm{c}}$, E. Ozmen ${ }^{\mathrm{d}}$, G. Ocakoglu ${ }^{\mathrm{e}}$, S. Bercin ${ }^{\mathrm{f}}$, D.A. Porter ${ }^{\mathrm{g}}$, A. Kutluhan $^{\mathrm{f}}$ \\ a Department of Radiology, Ataturk Training and Research Hospital, 06050 Ankara, Turkey \\ ${ }^{\mathrm{b}}$ National MR Research Center (UMRAM), Bilkent University, Bilkent, Ankara, Turkey \\ ' Department of Radiology, Dr. Abdurrahman Yurtaslan Oncology Hospital, Ankara Turkey \\ d Department of Radiology, Istanbul University, Cerrahpasa Medical School, Istanbul, Turkey \\ e Department of Biostatistics, Uludag University, Medical Faculty, Gorukle, Bursa, Turkey \\ f Otorhinolaryngology department, Yildırım Beyazıt University, Ataturk Training and Research Hospital, Ankara, Turkey \\ g Siemens AG, Healthcare Sector, Erlangen, Germany
}

\section{A R T I C L E I N F O}

Article history:

Available online 30 June 2017

\section{Keywords:}

Readout-segmented EPI

RESOLVE

Diffusion-weighted imaging

Cholesteatoma

Single-shot EPI

\begin{abstract}
A B S T R A C T
The purpose of this study is to evaluate the impact of RS-EPI-DWI in the detection of cholesteatoma and to compare with single-shot echo-planar DWI (SS-EPI-DWI). Diffusion-weighted and apparent diffusioncoefficient $(A D C)$ images were obtained using RS-EPI and SS-EPI techniques in 30 patients. Presence of cholesteatoma ( 3 point scale), amount of artefacts (4 point scale), visibility (4 point scale), and ADC values of the lesions were assessed. The results of both techniques were compared with each other and gold-standard (GS) test results. Lesion visibility and presence of artefact scores of RS-EPI-DWI group were significantly different from those of the SS-EPI group. RS-EPI-DWI images had fewer artefacts and higher visibility scores. The sensitivity, specificity, negative/positive-predictive, and overall-agreement values of RS-EPI-DWI technique were $100 \%, 78 \%, 100 \%, 74 \%$, and $87 \%$; respectively. These values for SS-EPI-DWI technique were 91\%,60\%, 88\%,67\%, and 75\%; respectively. Also, these values were higher on axial plane than coronal plane images for ADC measurements. Based on gold-standard test findings, agreement values were good $(\kappa=0.74)$ for RS-EPI-DWI and moderate for SS-EP-DWI $(\kappa=0.50)$ techniques $(P<0.001$ for both). The RS-EPI-DWI technique allows a higher spatial-resolution and this technique is less susceptible to artefacts when compared with SS-EPI technique.
\end{abstract}

(C) 2017 Elsevier Masson SAS. All rights reserved.

\section{Introduction}

Surgical treatment of the cholesteatoma is more functional and convenient when it is diagnosed earlier [1-3]. The surgery is less successful when the diagnosis is not clear and/or the size/location of the lesion is not exactly known [1-5]. Otoscopy, oto-endoscopy and microscopy are some of the clinical procedures used for the diagnosis of cholesteatoma [1]. High-resolution computed tomography (HRCT) and diffusion-weighted imaging (DWI) are the most commonly used imaging techniques for the diagnosis of the cholesteatoma [3-6].

* Corresponding author at: Department of Radiology, Ataturk Training and Research Hospital, 06050 Ankara, Turkey.

E-mail addresses: droktayalgin@gmail.com (O. Algin),

dr.hasanaydin@hotmail.com (H. Aydın), evrimkilicdr@gmail.com (E. Ozmen), gocakoglu@gmail.com (G. Ocakoglu), turkradyolojimyk@gmail.com (S. Bercin), david.a.porter@siemens.com (D.A. Porter), ahkutluhan@hotmail.com (A. Kutluhan).
Single-shot echo-planar imaging (SS-EPI) based DWI has several weaknesses including limited spatial resolution and geometric distortion due to the susceptibility artefacts within the temporal bones [7]. Geometric distortion may lead to false negative or positive results by preventing the optimal evaluation of middle ear cavity and those artefacts are more prominent at 3 tesla $(\mathrm{T})$ devices $[2,8]$. New techniques have been developed to avoid these artefacts including multi-shot echo-planar imaging (EPI), non-EPI or periodically rotated overlapping parallel lines with enhancedreconstruction (PROPELLER) techniques $[2,7,9,10]$.

Readout-segmented echo-planar imaging based (RS-EPI) technique was introduced by Porter et al. [8]. This method provides images with less artefacts and higher resolution for the evaluation of brain, brain stem and the breast [11-13]. However, to the best of our knowledge, its effectiveness in the evaluation of middle-ear cholesteatoma has not been established, yet.

The aim of this preliminary prospective research study was to evaluate the efficacy of DWI obtained with RS-EPI technique at $3 \mathrm{~T}$ MR device in detecting cholesteatoma and to compare this technique with SS-EPI method. 


\section{Materials and methods}

\section{Study population and MR exams}

This study was approved by the institutional review board and written consent was obtained from each of the patients. For the study, 49 patients with the prediagnosis of cholesteatoma at otoscopy or HRCT were evaluated using $3 \mathrm{~T}$ MR machine (TimTrio, Siemens, Erlangen, Germany) during three-year period with multi-channel birdcage head coil. All cases were scanned in a supine position without pre-imaging preparation. The same MR protocol was used for all subjects. Pregnant women and the patients with cardiac pacemaker/metallic implant or claustrophobia were excluded from the study. Moreover, 19 patients in whom cholesteatoma was detected by MR imaging at $3 \mathrm{~T}$, were excluded since they did not accept surgical treatment or had co-morbidities that made the surgery impossible. As a result, the final patient number included in the study was 30 (median age: 42 [12-65 years]; 11 male, 19 female). Median ages were 48 (13-65) and 42 (12-63) years for males and females, respectively. Nine (30\%) of the patients included in the study had previous ear surgery for cholesteatoma.

After sagittal plane three-dimensional (3D) T1 weighted (W) and T2 W sequences with isotropic voxel sizes $\left(<1 \mathrm{~mm}^{3}\right)$, axial/coronal planes SS-EPI and RS-EPI sequences were performed with comparable imaging parameters (Table 1). SS-EPI and RSEPI techniques were used for obtaining images from the same anatomic regions with the same image thickness and arranged to be parallel to the line that connected the internal acoustic channels. Images were acquired with $B$ values of $0,500,1000 \mathrm{~s} / \mathrm{mm}^{2}$ and $\mathrm{ADC}$ images were calculated using software provided by the manufacturer. Sequence parameters and details of the MRI protocol are presented in Table 1. Total imaging time for the $3 \mathrm{~T} \mathrm{MR}$ imaging was approximately 30 minutes.

\section{Evaluation of patients' data}

All of the MR images were transferred and evaluated by two radiologists with long-standing experience ( $>9$ years) in temporal bone imaging, who were blinded to the patients' clinical information, previous imaging data, and/or surgical findings, using a Leonardo workstation (Siemens, Erlangen, Germany).

The first session was designed as the SS-EPI session where all the images were obtained excluding the RS-EPI images, evaluated and scored regarding the presence of cholesteatoma ( 3 point scale) and lesion visibility (4 point scale) as explained below.

\section{Cholesteatoma scoring}

Score 0: normal middle ear cavity (negative result).

Score 1: suspicious findings (indefinite).

Score 2: definitive cholesteatoma (positive result).

\section{Lesion visibility-conspicuity scores}

Score 0: lesion is not visible.

Score 1: suspicious lesion(s) is/are exist but cannot be discriminated from the surrounding tissue or is/are not seen clearly (inadequate or poor visibility).

Score 2: discrimination is good.

Score 3: excellent lesion conspicuity or visibility.

In the second session (the RS-EPI session); the scoring procedure mentioned above was performed also for RS-EPI images and $\mathrm{T} 1 \mathrm{~W} / \mathrm{T} 2 \mathrm{~W}$ images. In this session, the SS-EPI sequences were not evaluated. To avoid bias that might be originated from memory effects, none of the scoring sessions had a similar patient order whilst evaluating.

The diagnosis of cholesteatoma on MRI was based on the presence of increased signal intensity on $b$ value $=1000 \mathrm{~s} / \mathrm{mm}^{2}$, decreased signal intensity on ADC and a soft-tissue mass seen on 3D T1 W or T2 W images without evidence of other pathology (such as cholesterol granuloma). The intensity of lesion was decided by comparing with the intensity of cerebellum on both b1000 and ADC images. In case of the existence of a hyperintense lesion on b1000 images, we measured the corresponding ADC values in a selected region of interest (ROI). For each lesion, the ROI was defined to encompass the maximal amount of the lesion without exceeding the lesion's margins. The size of the lesion was measured in the greatest diameters on axial and coronal b1000 images.

In the sessions mentioned above; the artefacts on DWI and ADC images were also evaluated. During these assessments of both EPI techniques, the existence and amount of the motion artefacts, geometric distortions, and image blurring adjacent to the middle ear cavity were scored visually as described below.

\section{Artefact scoring}

Score 0: unacceptable (DWI quality severely deteriorated by artefacts).

Score 1: acceptable (there are prominent artefacts however images can be evaluated properly).

Score 2: good (there are few artefacts and those artefacts do not prevent image evaluation).

Score 3: excellent (artefact-free imaging).

For all scorings, the final decisions were obtained with consensus.

All patients who had cholesteatoma on MRI were operated within 3 months following $3 \mathrm{~T}$ MR examination. The surgical findings were obtained from the surgical reports of two different experienced otorhinolaryngology teams. Surgical results were accepted as gold-standard (GS) test results. However, most of the patients who did not have cholesteatoma on $3 \mathrm{~T}$ MR examinations had final diagnosis with clinical, temporal-bone CT, contrastmaterial enhanced MRI with delayed imaging, and/or follow-up (mean length of follow-up is 41-month) findings by the same two radiologists and otorhinolaryngology teams with consensus, since those patients were not operated on. Surgical results or abovementioned final decisions were analysed and compared with SS-EPI and RS-EPI findings.

\section{Statistical analysis}

Continuous variables were expressed with median (minimum-maximum) values. Agreements between visibility, artefact, presence of cholesteatoma scores and ADC values were evaluated by using Cohen's kappa and intra-class correlation coefficient (ICC). Between-group comparisons of RS-EPI and SSEPI values were performed by using the Mann Whitney U-test. The diagnostic accuracy of the SS-EPI and the RS-EPI sequences was described by sensitivity, specificity and positive $\&$ negative predictive value (PPV \& NPV). Cut-off points of ADC values were determined by using ROC (receiver operating curve) analysis. Area under the curve, sensitivity, specificity, positive and negative predictive values were also reported as p-values. In all statistical analyses, the level of significance was set to $\alpha=0.05$. Statistical analyses were performed with SPSS software version 21.0 (Chicago, IL, USA).

\section{Results}

There was no significant difference between males and females in terms of age $(P=0.226)$. According to the GS test results, cholesteatoma was detected in 21 patients. Two of these patients have bilateral cholesteatomas (these patients had surgery done on both ears), while unilateral disease was existed in the other 19 patients (Figs. 1 and 2). Thus, cholesteatoma was detected in 23 
Table 1

3 tesla MRI protocol of the study.

\begin{tabular}{|c|c|c|c|c|c|}
\hline Sequences/parameters & STIR & 3D-MPRAGE & 3D-SPACE & SS-EPI & RS-EPI \\
\hline $\mathrm{TR} / \mathrm{TE}$ (ms) & $5100 / 81$ & $2100 / 2.36$ & $3000 / 423$ & $6500 / 111$ & $5000 / 91-124$ \\
\hline $\mathrm{TI}(\mathrm{ms})$ & 150 & 1000 & - & - & - \\
\hline Slice thickness (mm) & 3 & 0.66 & 0.78 & 3 & 3 \\
\hline $\mathrm{FOV}(\mathrm{mm})$ & $180 \times 180$ & $210 \times 210$ & $200 \times 200$ & $218 \times 218$ & $236 \times 236$ \\
\hline Acquisition time (min.) & 2 & 6 & 5 & 3 & 5 \\
\hline NEX & 2 & 1 & 1 & 4 & 1 \\
\hline Number of slices & 16 & 256 & 208 & 30 & 30 \\
\hline Flip angle $\left(^{\circ}\right)$ & 120 & 8 & Variant & 180 & 180 \\
\hline Imaging plane & Axial & Sagittal & Sagittal & Axial-coronal & Axial-coronal \\
\hline Distance factor (\%) & 10 & - & - & 20 & 20 \\
\hline PAT factor & 2 & 2 & 2 & 2 & 2 \\
\hline PAT mode & GRAPPA & GRAPPA & GRAPPA & GRAPPA & GRAPPA \\
\hline$B$ values & - & - & - & $0,500,1000$ & $0,500,1000$ \\
\hline Fat-suppression & - & + & - & + & + \\
\hline Base resolution & 384 & 320 & 256 & 192 & 160 \\
\hline Phase resolution (\%) & 80 & 100 & 100 & 100 & 100 \\
\hline
\end{tabular}

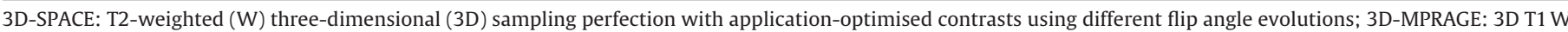

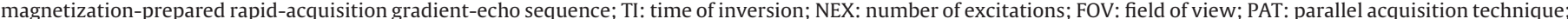

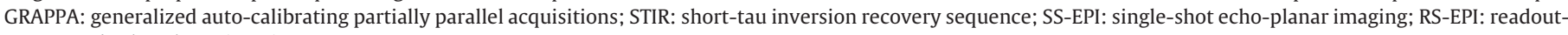
segmented echo-planar imaging.
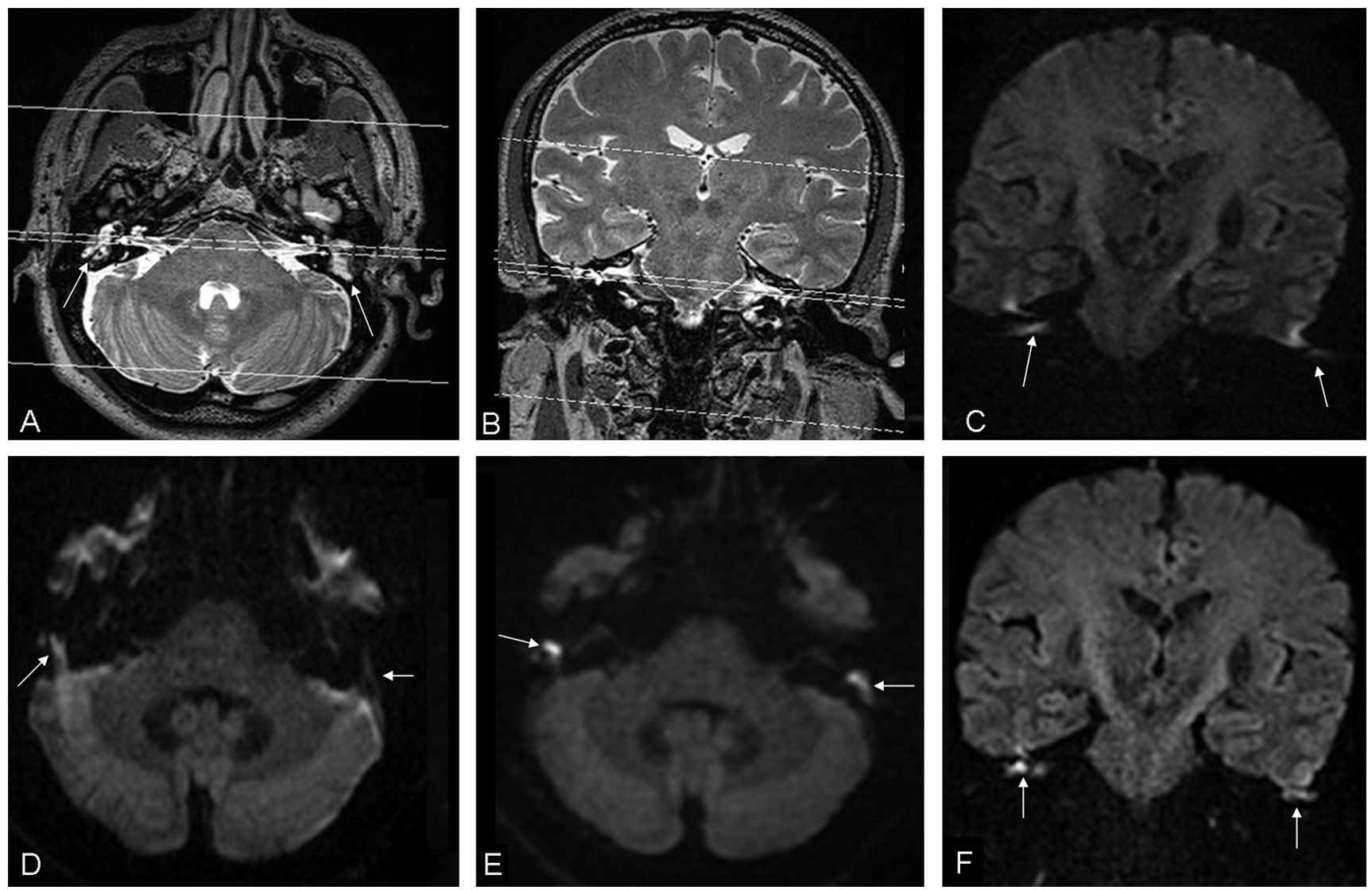

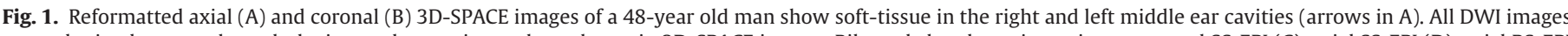

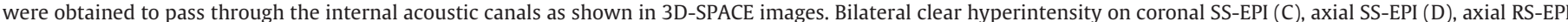

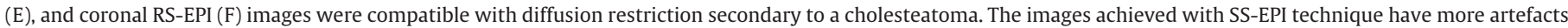
than the images obtained with RS-EPI technique (arrows). The lesion on the left side could not visualize clearly on SS-EPI images (arrows).

ears. The lesion was hypointense in 13 ears (57\%), isointense in nine ears (39\%) and hyperintense in one ear (4\%) on STIR images. In the remaining nine patients, cholesterol granuloma (1 patient) and chronic otitis media (8 patients) were present. The median dimension of the lesions at the middle ear cavity was $8 \mathrm{~mm}(2-29 \mathrm{~mm})$.

There was a mild coherence between the presence of cholesteatoma scores of RS-EPI and SS-EPI images (Table 2). No correlation was detected between lesion visibility and presence of
Table 2

Agreement values between the parameters.

\begin{tabular}{llll}
\hline & ICC & $\kappa$ & $P$-value \\
\hline RS-EPI vs. SS-EPI (artefact scores) & - & -0.086 & 0.060 \\
EPI vs. SS-EPI (visibility scores) & - & -0.064 & 0.278 \\
RS-EPI vs. SS-EPI (coronal plane ADC values) & 0.723 & - & $<0.001$ \\
RS-EPI vs. SS-EPI (axial plane ADC values) & 0.976 & - & $<0.001$ \\
\hline
\end{tabular}

ICC: intra-class correlation coefficient, к: kappa coefficient. 

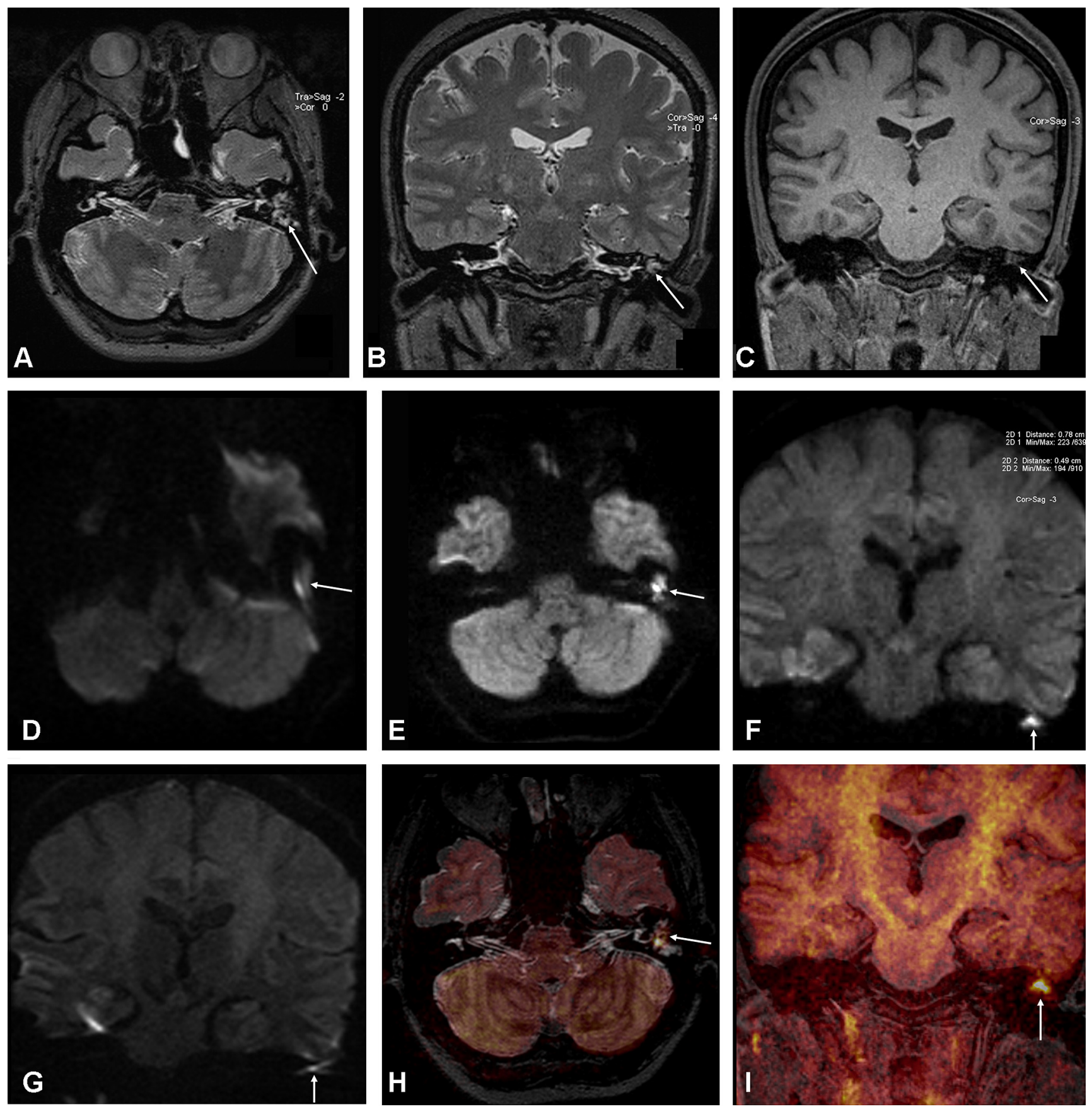

Fig. 2. Reformatted axial-coronal T2 W 3D-SPACE (A, B), and coronal MPRAGE (C) images of a 30-year-old female show soft-tissue that filled left middle ear (arrows). A hyperintense lesion representing cholesteatoma with the size of $6 \times 7 \mathrm{~mm}$ is seen on DWI (arrows, D-G). More artefacts exist on axial and coronal SS-EPI images (D, G) when compared with axial and coronal RS-EPI images (E, F). It is also demonstrated that the lesion contour can be visualized more clearly on RS-EPI images. The localization and the relation with adjacent structures of cholesteatoma can be evaluated better on axial (T2 W 3D-SPACE plus DWI, H) and coronal (MPRAGE plus DWI, I) fused images (arrows).

Table 3

Numbers visibility and presence of artefact scores of RS-EPI and SS-EPI groups.

\begin{tabular}{|c|c|c|c|c|c|c|}
\hline & 0 & 1 & 2 & 3 & $\mathrm{~N}^{\mathrm{a}}$ & Total \\
\hline \multicolumn{7}{|c|}{ Visibility scores } \\
\hline RS-EPI & - & $1(2 \%)$ & $17(28 \%)$ & $25(42 \%)$ & $17(28 \%)$ & $60(100 \%)$ \\
\hline SS-EPI & $1(2 \%)$ & $18(30 \%)$ & $24(40 \%)$ & - & $17(28 \%)$ & 60 (100\%) \\
\hline \multicolumn{7}{|c|}{ Artefact scores } \\
\hline RS-EPI & $1(2 \%)$ & $1(2 \%)$ & $17(28 \%)$ & $24(40 \%)$ & $17(28 \%)$ & $60(100 \%)$ \\
\hline SS-EPI & $1(2 \%)$ & $29(48 \%)$ & $13(22 \%)$ & - & $17(28 \%)$ & $60(100 \%)$ \\
\hline
\end{tabular}

\footnotetext{
a $\mathrm{N}$ : completely normal ears are not scored.
} 

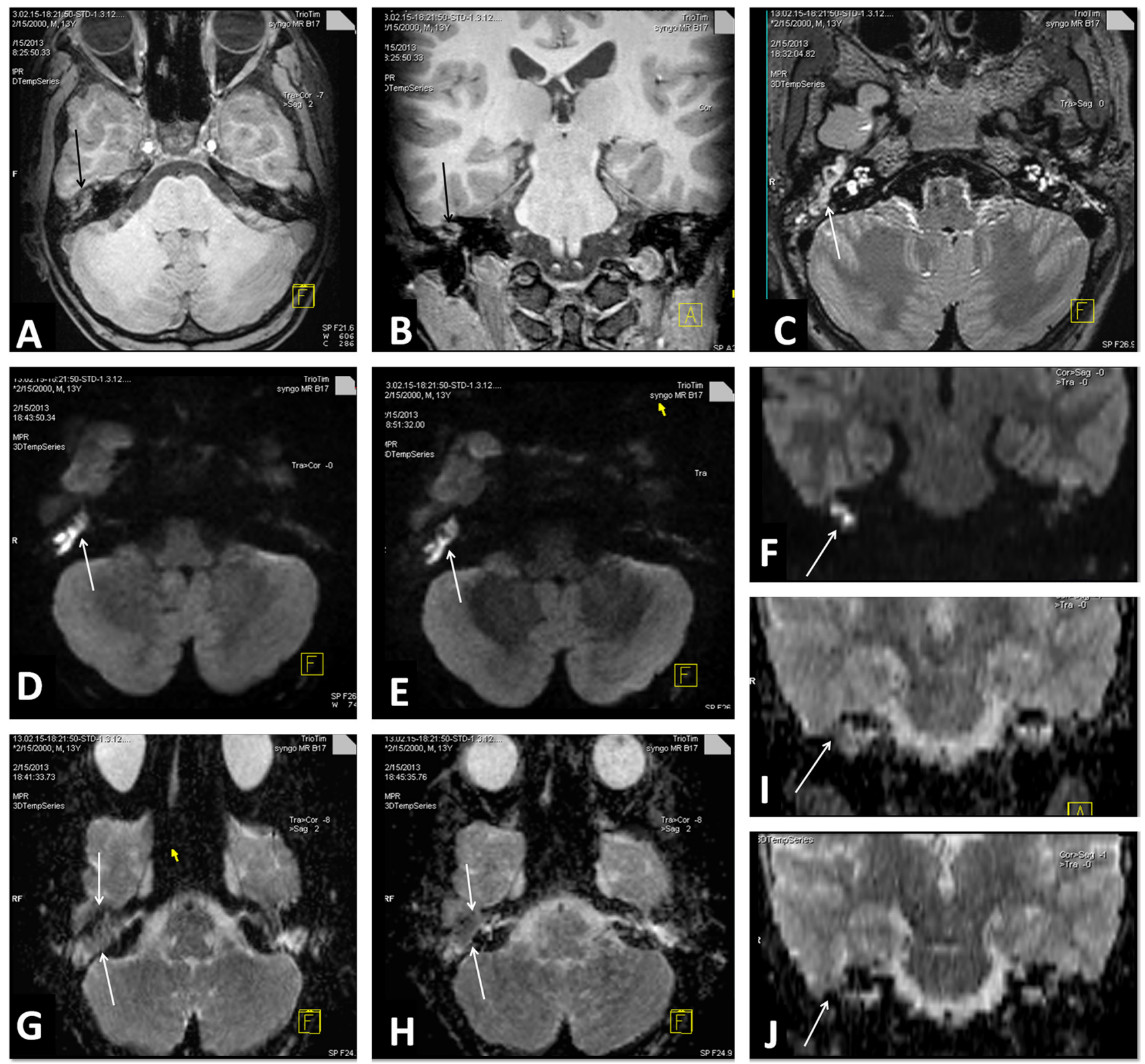

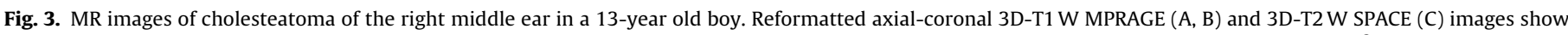

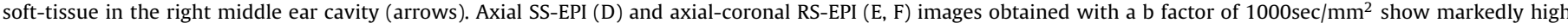

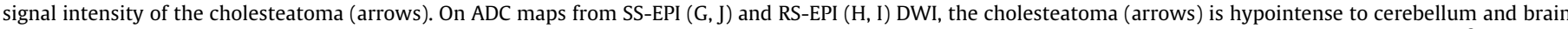

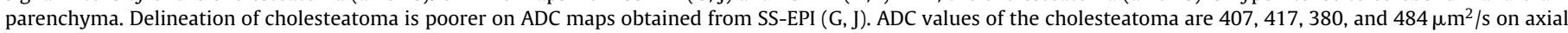
RS-EPI, coronal RS-EPI, axial SS-EPI, and coronal SS-EPI maps, respectively.

artefact scores of RS-EPI and SS-EPI groups $(P>0.05)$ (Table 2$)$. Artefact scores of RS-EPI images were lower than those of SS-EPI images (Table 3). In addition, the visibility scores of the lesions on RS-EPI images were higher than those on SS-EPI images (Table 3) (Fig. 3).

The comparison between GS tests and ADC values are presented in Table 4. ADC values of GS $=0$ (absence of cholesteatoma) group was significantly higher when compared with the ADC values of $\mathrm{GS}=1$ (presence of cholesteatoma) group $(P<0.05$, Table 4$)$.

The sensitivity, specificity, NPV and PPV values of ADC measurements obtained with RS-EPI and SS-EPI techniques for different cut-off values are given in Table 5 . Area under curve (AUC) values was tended to be higher in axial plane measurements than those in coronal plane measurements (Table 5).

During the statistical comparison of RS-EPI and SS-EPI with GS test findings in terms of detecting cholesteatoma, we noticed that the RS-EPI technique was more comparable with the GS test results
( $\kappa=0.74, P<0.001$ for RS-EPI; $\kappa=0.5, P=0.001$ for SS-EPI) (Table 6 ) (Fig. 1). Using the surgical outcome as the gold standard, the sensitivity, specificity, PPV and NPV of RS-EPI were higher than SS-EPI (100\%, $78 \%, 100 \%$, and $74 \%$ for RS-EPI versus $91 \%, 60 \%, 88 \%$, and $67 \%$ for SS-EPI-DWI, respectively) (Table 6).

\section{Discussion}

Cholesteatoma is a benign condition, which may lead to serious complications including hearing loss, labyrinthine fistula and vestibular dysfunction unless it is treated in time. Early diagnosis and treatment may reduce the complication rates and provide a better prognosis. HRCT is the most commonly used technique for scanning temporal bone. Although HRCT can be useful for the diagnosis of the patients without middle-ear surgery history, it is often 
Table 4

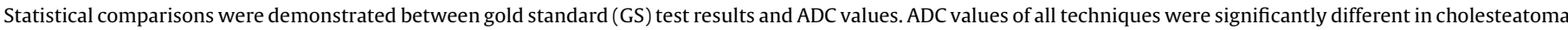
and non-cholesteatoma groups.

\begin{tabular}{|c|c|c|c|}
\hline & $\mathrm{GS}=0$ (absence of cholesteatoma) & $\mathrm{GS}=1$ (presence of cholesteatoma) & $P$-value \\
\hline & Median (min.-max.) & Median (min.-max.) & \\
\hline RS-EPI ADC (axial) & $873(398-2200) \mu \mathrm{m}^{2} / \mathrm{s}$ & $500(285-1178) \mu \mathrm{m}^{2} / \mathrm{s}$ & 0.003 \\
\hline RS-EPI ADC (coronal) & $926(415-2200) \mu \mathrm{m}^{2} / \mathrm{s}$ & $511(400-1281) \mu \mathrm{m}^{2} / \mathrm{s}$ & 0.023 \\
\hline SS-EPI ADC (axial) & $960(491-2100) \mu \mathrm{m}^{2} / \mathrm{s}$ & $500(285-1179) \mu \mathrm{m}^{2} / \mathrm{s}$ & 0.001 \\
\hline SS-EPI ADC (coronal) & $920(600-1127) \mu \mathrm{m}^{2} / \mathrm{s}$ & $503(320-1325) \mu \mathrm{m}^{2} / \mathrm{s}$ & 0.003 \\
\hline
\end{tabular}

Table 5

Receiver operating curve (ROC) analysis results of ADC measurements.

\begin{tabular}{|c|c|c|c|c|c|c|c|}
\hline & AUC & $P$-value & Cut-off value & Sensitivity & Specificity & PPV & NPV \\
\hline RS-EPI ADC (axial) & 0.83 & $<0.001$ & $\leq 521$ & 56.52 & 90.91 & 92.9 & 50 \\
\hline SS-EPI ADC (axial) & 0.86 & $<0.001$ & $\leq 500$ & 56.52 & 90 & 92.9 & 47.4 \\
\hline SS-EPI ADC (coronal) & 0.83 & $<0.001$ & $\leq 503$ & 52.38 & 100 & 100 & 50 \\
\hline RS-EPI ADC (coronal) & 0.81 & $<0.001$ & $\leq 511$ & 52.17 & 90.91 & 92.3 & 47.6 \\
\hline RS-EPI ADC (axial) & 0.83 & $<0.001$ & $\leq 700$ & 82.61 & 63.64 & 82.6 & 63.6 \\
\hline SS-EPI ADC (axial) & 0.85 & $<0.001$ & $\leq 705$ & 86.96 & 60.00 & 83.3 & 66.7 \\
\hline SS-EPI ADC (coronal) & 0.83 & $<0.001$ & $\leq 706$ & 71.43 & 60.00 & 78.9 & 50.0 \\
\hline RS-EPI ADC (coronal) & 0.81 & $<0.001$ & $\leq 700$ & 73.91 & 63.64 & 81.0 & 53.8 \\
\hline
\end{tabular}

AUC: area under curve; PPV: positive predictive value; NPV: negative predictive value.

insufficient for the diagnosis of small sized cholesteatoma lesions (especially in patients with middle-ear surgery history) [14].

HRCT and DWI are usually combined for the diagnosis of cholesteatoma in daily routine practice. Although cholesteatomas show high signal intensity, granulation/fibrous tissue, cholesterol granuloma and serous fluid show low signal intensity on DWI [15]. Delayed phase gadolinium enhanced T1 W imaging can be used for the diagnosis of cholesteatoma; however there are conflicting data about this method in the literature. Although some authors suggested that delayed phase post-contrast imaging is useful, others indicated that delayed phase post-contrast imaging could not provide additive information $[9,16]$. These conflicting results were believed to be related with the dimension of cholesteatoma [16]. Delayed phase post-contrast T1 W imaging ( 45 minutes after intravenous gadolinium administration) has some disadvantages including increased cost, the scanning time and risk of nephrogenic systemic fibrosis [14,17]. In addition, some false positive results could be seen at delayed phase post-contrast $\mathrm{T} 1 \mathrm{~W}$ imaging such as retained inflammatory secretions, which may simulate a nonenhancing cholesteatoma [18].

In cholesteatoma, patients' geometric distortions and other artefacts are often severe on SS-EPI images of temporal bone especially at 3 T MR units [19]. The long duration of readout and 'low bandwidth value per pixel in the phase-encoding direction' are among the reasons of this condition [19]. Although many techniques (e.g. spin-echo based sequences or PROPELLER technique) were experimented for diminishing these artefacts, there are several limitations for each technique such as low resolution or long imaging time $[2,4-7,9,10]$.

RS-EPI-DWI is a novel technique and not reported for imaging of temporal bone in the literature. The basic methodology for

Table 6

Based on gold-standard test findings; sensitivity, specificity, NPV, PPV values, overall-agreement and K statistic results of RS-EPI and SS-EPI techniques.

\begin{tabular}{lll}
\hline & RS-EPI & SS-EPI \\
\hline Sensitivity (\%) & 100 & 91 \\
Specificity (\%) & 78 & 60 \\
NPV (\%) & 100 & 88 \\
PPV (\%) & 74 & 67 \\
Overall agreement (\%) & 87 & 75 \\
$K$ value & 0.74 & 0.50 \\
$P$-value & $<0.001$ & $<0.001$ \\
\hline
\end{tabular}

the RS-EPI technique was introduced by Porter et al. [8]. Our preliminary study showed that RS-EPI method could provide higher spatial-resolution and lesion-visibility with less distortion and artefacts than SS-EPI technique, which is relevant with other studies [19-21]. Analysing the presence of cholesteatoma scores of our study showed that RS-EPI technique differs significantly from SSEPI technique and could detect cholesteatoma in higher number of patients. Hence, RS-EPI technique can supply a more effective evaluation for cholesteatomas.

Although the sensitivity of SS-EPI technique is relatively high for detecting the lesions more than $5 \mathrm{~mm}$, the effectiveness of this technique is low in detecting lesions that are less than $5 \mathrm{~mm}$ [18]. We could not visualize the lesion clearly and could not measure ADC value in coronal plane of SS-EPI in three patients and in axial plane of SS-EPI in one patient among our patient group. Two out of 4 patients that mentioned above were operated for cholesteatoma previously. The mean lesion size was $7 \mathrm{~mm}$ (range 5-9.5) and the lesions could be visualised (clearly depicted) and ADC measurements could be performed, with RS-EPI technique in those patients. RS-EPI method seems to be more useful in detection of small lesions and/or in evaluation of postoperative patients.

According to the literature, the ADC values of cholesteatomas $\left(700-1100 \mu \mathrm{m}^{2} / \mathrm{s}\right.$ ) have been reported to be equal or less than the brain parenchyma while those values of abscess $\left(400-570 \mu \mathrm{m}^{2} / \mathrm{s}\right)$ have been reported to be significantly lower than the brain parenchyma $[22,23]$. However there is not a reported cut-off value related to ADC values in the literature. Similarly, a certain cut-off value could not be indicated in our study as well. However, our findings are compatible with the literature data $[3,16,18,22-24]$.

Another important outcome of our study was that ADC measurements from axial images had better AUC, NPV, PPV, sensitivity and specificity values when compared with the coronal image ADC measurements. This may be explained by the fact that the coronal plane DWI is more sensitive to the susceptibility artefacts. Thus, DWI images of middle ear cavity should be obtained in the axial plane. We think that performing $\mathrm{T} 1$ and $\mathrm{T} 2 \mathrm{~W}$ images in addition to RS-EPI is necessary for accurate detection of localization \& extension of the lesions and detection of other pathologic conditions of the ears and/or cranium (such as fistulas, masses or abscess) $[14,17]$. As we have experienced in our study, 3D isotropic (voxel sizes $<1 \mathrm{~mm}$ ) acquisition of $\mathrm{T} 1 \mathrm{~W}$ and $\mathrm{T} 2 \mathrm{~W}$ sequences might be useful for evaluation of whole cranium simultaneously. An optimised and robust MR examination can be achieved only in 15 minutes 
by adding axial RS-EPI DWI images to sagittal 3D T1 W and T2 W sequences (Table 1 ).

The possibility of missing small size cholesteatoma due to nonisotropic acquisition of DWI or relatively thick slices has been reported previously [14,24]. Usually, mural cholesteatomas are not visible on DWI [18]. We detected relatively minimal susceptibility related distortion (especially on coronal plane images) of the bone and air transition zone in some patients although it was significantly less on RS-EPI images when compared with the SS-EPI technique. Mostly those artefacts were not detrimental for the diagnosis. However, new and comprehensive studies are needed for the development of a completely artefact-free DWI technique.

As a limitation, we did not compare RS-EPI-DWI with a nonEPI sequence, which is one of current imaging techniques due to time restriction of the MRI acquisitions [25]. A comparison between RS-EPI-DWI and a non-EPI sequence would be welcome. Also, this study only includes patients with a prediagnosis of cholesteatoma. Therefore, this study lacks critical information of the positive predictive value of the test. Neither of the patients with negative test results where operated. Since clinical and imaging follow-up (range: 1-5 years) is less reliable. This factor is a risk of an underestimation of the false negative results. Finally, there is a strong possibility of incorporation bias and reader order bias. These are difficult to avoid.

In conclusion, DWI with RS-EPI, which is a relatively artefactfree technique and has a higher resolution than the SS-EPI method, provides a more effective assessment for cholesteatomas. Also, the RS-EPI technique seems to be more efficient in the detection of small lesions and/or in the evaluation of postoperative patients.

\section{Disclosure of interest}

DAP is an employee and shareholder of Siemens AG. Two US patents have been registered by DAP for the diffusion-weighted readout-segmented EPI sequence used in this paper and are assigned to Siemens AG. The method is also marketed by Siemens AG as a commercial product using the name 'RESOLVE'.

\section{Acknowledgement}

We gratefully acknowledge Dr. Elcin Zan and Dr. Ali Güvey for their valuable contributions.

\section{References}

[1] Aikele P, Kittner T, Offergeld $C$, et al. Diffusion-weighted MR imaging of cholesteatoma in pediatric and adult patients who have undergone middle ear surgery. AJR Am J Roentgenol 2003;181:261-5.

[2] Ilica AT, Hidir Y, Bulakbaşi N, et al. HASTE diffusion-weighted MRI for the reliable detection of cholesteatoma. Diagn Interv Radiol 2012;18:153-8.

[3] Khemani S, Singh A, Lingam RK, Kalan A. Imaging of postoperative middle ear cholesteatoma. Clin Radiol 2011;66:760-7.

[4] Aarts MC, Rovers MM, van der Veen EL, et al. The diagnostic value of diffusionweighted magnetic resonance imaging in detecting a residual cholesteatoma. Otolaryngol Head Neck Surg 2010;143:12-6.
[5] Cimsit NC, Cimsit C, Baysal B, et al. Diffusion-weighted MR imaging in postoperative follow-up: reliability for detection of recurrent cholesteatoma. Eur J Radiol 2010;74:121-3.

[6] De Foer B, Vercruysse JP, Pilet B, et al. Single-shot, turbo spin-echo, diffusionweighted imaging versus spin-echo-planar, diffusion-weighted imaging in the detection of acquired middle ear cholesteatoma. AJNR Am J Neuroradiol 2006;27:1480-2.

[7] Yamashita K, Yoshiura T, Hiwatashi A, et al. Detection of middle ear cholesteatoma by diffusion-weighted MR imaging: multishot echo-planar imaging compared with single-shot echo-planar imaging. AJNR Am J Neuroradiol 2011;32:1915-8.

[8] Porter DA, Heidemann RM. High resolution diffusion-weighted imaging using readout-segmented echo-planar imaging, parallel imaging and a two-dimensional navigator-based reacquisition. Magn Reson Med 2009;62: 468-75.

[9] De Foer B, Vercruysse JP, Bernaerts A, et al. Middle ear cholesteatoma: non-echo-planar diffusion-weighted MR imaging versus delayed gadoliniumenhanced T1-weighted MR imaging - value in detection. Radiology 2010;255:866-72.

[10] Dremmen MH, Hofman PA, Hof JR, Stokroos RJ, Postma AA. The diagnostic accuracy of non-echo-planar diffusion-weighted imaging in the detection of residual and/or recurrent cholesteatoma of the temporal bone. AJNR Am J Neuroradiol 2012;33:439-44.

[11] Holdsworth SJ, Yeom K, Skare S, et al. Clinical application of readoutsegmented- echo-planar imaging for diffusion-weighted imaging in pediatric brain. AJNR Am J Neuroradiol 2011;32:1274-9.

[12] Bogner W, Pinker-Domenig K, Bickel H, et al. Readout-segmented echo-planar imaging improves the diagnostic performance of diffusion-weighted MR breast examinations at 3.0 T. Radiology 2012;263:64-76.

[13] Naganawa S, Yamazaki M, Kawai H, et al. Anatomical details of the brainstem and cranial nerves visualized by high resolution readout-segmented multi-shot echo-planar diffusion-weighted images using unidirectional MPG at 3 T. Magn Reson Med Sci 2011;10:269-75.

[14] Vaid S, Kamble Y, Vaid N, et al. Role of magnetic resonance imaging in cholesteatoma: the Indian experience. Indian J Otolaryngol Head Neck Surg 2013;65:485-92.

[15] Dubrulle F, Souillard R, Chechin D, et al. Diffusion-weighted MR imaging sequence in the detection of postoperative recurrent cholesteatoma. Radiology 2006;238:604-10

[16] Venail F, Bonafe A, Poirrier V, Mondain M, Uziel A. Comparison of echoplanar diffusion-weighted imaging and delayed postcontrast T1-weighted MR imaging for the detection of residual cholesteatoma. AJNR Am J Neuroradiol 2008;29:1363-8.

[17] De Foer B, Vercruysse JP, Spaepen M, et al. Diffusion-weighted magnetic resonance imaging of the temporal bone. Neuroradiology 2010;52:785-807.

[18] Más-Estellés F, Mateos-Fernández M, Carrascosa-Bisquert B, et al. Contemporary non-echo-planar diffusion-weighted imaging of middle ear cholesteatomas. Radiographics 2012;32:1197-213.

[19] Morelli JN, Saettele MR, Rangaswamy RA, et al. Echo planar diffusion-weighted imaging: possibilities and considerations with 12- and 32-channel head coils. J Clin Imaging Sci 2012;2:31.

[20] Holdsworth SJ, Skare S, Newbould RD, et al. Readout-segmented EPI for rapid high resolution diffusion imaging at 3 T. Eur J Radiol 2008;65:36-46.

[21] Holdsworth SJ, Skare S, Newbould RD, Bammer R. Robust GRAPPAaccelerated diffusion-weighted readout-segmented (RS)-EPI. Magn Reson Med 2009;62:1629-40.

[22] Thiriat S, Riehm S, Kremer S, Martin E, Veillon F. Apparent diffusion coefficient values of middle ear cholesteatoma differ from abscess and cholesteatoma admixed infection. AJNR Am J Neuroradiol 2009;30:1123-6.

[23] Karandikar A, Loke SC, Goh J, Yeo SB, Tan TY. Evaluation of cholesteatoma: our experience with DW propeller imaging. Acta Radiol 2015;56:1108-12.

[24] Pennanéach A, Garetier M, Ollivier M, Ognard J, Marianowski R, Meriot P. Diagnostic accuracy of diffusion-weighted MR imaging versus delayed gadolinium enhanced T1-weighted imaging in middle ear recurrent cholesteatoma: a retrospective study of 39 patients. J Neuroradiol 2016;43:148-54.

[25] Lincot J, Veillon F, Riehm S, et al. Middle ear cholesteatoma: compared diagnostic performances of two incremental MRI protocols including non-echo planar diffusion-weighted imaging acquired on $3 \mathrm{~T}$ and $1.5 \mathrm{~T}$ scanners. J Neuroradiol 2015;42:193-201. 